\title{
A Histerossonografia na Avaliação da Cavidade Uterina Alterada em Pacientes Pós-Menopausadas
}

Autor: Benito Pio Vitório Ceccato Júnior

Orientador: Prof. Dr. Victor Hugo de Melo

Dissertação de Mestrado apresentada ao Curso de Pós-graduação em Ginecologia e Obstetrícia da Faculdade de Medicina da Universidade Federal de Minas Gerais em $1^{\circ}$ de março de 2002.

Objetivos: Avaliar a acurácia diagnóstica da histerossonografia como método de avaliação da cavidade uterina em pacientes pós-menopausadas com cavidade uterina alterada à ultrasonografia endovaginal convencional. Métodos: Este estudo consistiu na avaliação de 99 pacientes pós-menopausadas com cavidade uterina anormal à ultra-sonografia endovaginal convencional, caracterizada por espessura endometrial maior ou igual a $5 \mathrm{~mm}$ em pacientes sem terapia de reposição hormonal, ou espessura endometrial maior ou igual a $8 \mathrm{~mm}$ em pacientes em terapia de reposição hormonal, com sangramento irregular. Estas pacientes foram submetidas à histerossonografia e, após, foram obtidas amostras para avaliação histopatológica por biópsia dirigida por histeroscopia em 92 pacientes, biópsia endometrial em quatro pacientes, e histerectomia em três pacientes. Os resultados da histerossonografia foram comparados com os resultados do exame histopatológico, considerado como padrão ouro.

Resultados: A histerossonografia teve altos niveis de especificidade no diagnóstico da cavidade uterina normal e atrófica $(97,5 \%$ e $97,8 \%)$, e baixa sensibilidade
(35\% e 25\%). Houve altos niveis de sensibilidade $(92,3 \%$ e $75,0 \%)$ e especificidade (94,1\% e $97,9 \%)$ em pólipos e miomas submucosos. Houve três casos de câncer de endométrio e a HSNG teve sensibilidade e especificidade de $100 \%$

Conclusões: A histerossonografia mostrou boa acurácia no diagnóstico de patologias focais (pólipos endometriais e miomas submucosos), com altos niveis de sensibilidade e especificidade. Houve três casos de câncer endometrial, e a histerossonografia diagnosticou corretamente todos eles. Mostrou também ser um método acurado para excluir anormalidades endometriais. Entretanto, nos casos de espessamento endometrial difuso, a acuidade é baixa, porque endométrios atróficos ou normais freqüentemente aparecem como tendo espessamento difuso à ultra-sonografia endovaginal e à histerossonografia. A histerossonografia não teve complicações durante e após o procedimento.

Palavras-chave: Menopausa. Endométrio: câncer. Endométrio: hiperplasia. Histerossonografia.

\section{Comportamento do Comprimento do Colo Uterino em Gestações Normais Avaliado pela Ultra- sonografia Transvaginal}

Aluno: Reginaldo Antonio de Oliveira Freitas Júnior

Orientador: Prof. Dr. Francisco Mauad Filho

Dissertação para obtenção do título de Mestre apresentada ao Departamento de Ginecologia e Obstetrícia da Faculdade de Medicina de Ribeirão Preto, em 16/7/2001.

Objetivo: construir uma curva de normalidade do comprimento cervical uterino avaliado por meio da ultrasonografia transvaginal, ao longo da gestação, estabelecendo parâmetros para comparação frente a gestações patológicas, sobretudo às implicadas em maior risco para o parto pré-termo.

Métodos: foi realizado um estudo prospectivo, longitudinal, no qual 82 gestantes hígidas foram acompanhadas desde o início da gestação, das quais 49 mantiveram o seguimento até o parto, sendo examinadas a cada intervalo de quatro semanas e agrupadas conforme a paridade, em nuliparas ou não-nulíparas.

Resultados: as médias de comprimento do colo uterino, bem como os percentis $5,10,25,50,75,90$ e 95, em função da idade gestacional, não diferiram significativamente entre os grupos estudados $(p>0.05)$. O comprimento cervical uterino diminui progressivamente ao longo da gestação normal, sendo esse encurtamento significativo após a 20 a semana de gravidez e mais expressivo após a $28^{\text {a }}$ semana gestacional $(\mathrm{p}<0.05)$. Conclusões: o padrão de comportamento do comprimento cervical uterino parece não diferir entre nuliparas e mulheres com um ou mais partos anteriores. Os valores numéricos da curva de normalidade do comprimento cervical uterino, em função da idade gestacional, refletem a variabilidade perante as características peculiares à amostra estudada, devendo, portanto, serem valorizados os parâmetros estabelecidos para a nossa população.

Palavras-chave: Comprimento do colo. Gravidez normal. Ultra-sonografia transvaginal. Prematuridade. Colo. do útero. 